\title{
Scattering of Sound by Internal Wave Currents: The Relation to Vertical Momentum Flux
}

\author{
W. MUNK AND P. WORCESTER \\ Scripps Institution of Oceanography, La Jolla, CA 92093 \\ F. ZACHARIASEN \\ California Institute of Technology, Pasadena, CA 91109
}

(Manuscript received 19 September 1980, in final form 8 January 1981)

\begin{abstract}
Internal waves scatter sound by two related perturbations: 1) those associated with vertical particle displacements $\zeta(x, y, z, t)$ in the presence of a vertical gradient of (potential) sound speed $\left(\delta c=\zeta \partial_{z} c_{p}\right)$; and 2) those associated with horizontal particle velocities $u(x, y, z, t)$. The combined fractional perturbation in propagation velocity is $\delta c / c+u / c$. The second term, generally neglected, introduces a nonreciprocity when source and receiver are interchanged. Nonreciprocity is expected to be relatively small except for transmission along a deep downward loop. The principal internal wave contribution to nonreciprocity is from inertial frequencies. The sum and difference of reciprocal travel times are a measure of $\zeta$ and $u$, respectively, along the ray path; the quadrature spectrum of reciprocal travel times is related by an integral equation to the spectrum of the momentum flux $\langle\dot{\zeta} u\rangle$. Precise measurements of nonreciprocity could provide an estimate of the vertical momentum flux in an internal wave field.
\end{abstract}

\section{Introduction}

In the absence of a velocity field the character of a sound transmission would be the same in all detail if source and receiver were interchanged. Any nonreciprocity is therefore evidence for a flow field, and contains some information concerning this flow field. Worcester's (1977) experiment of reciprocal transmission in a midocean environment revealed a high degree of nonreciprocity in short period (cph) fluctuations. In this paper we have asked whether these fluctuations can be accounted for by the particle velocities associated with internal waves, and whether one could derive oceanographically useful information in this manner. We have not succeeded in explaining the experimental results, but have found that the cross spectrum in oppositely directed acoustic travel times could provide a measure of the correlation between vertical and horizontal particle velocities, e.g., the vertical flux of horizontal momentum.

The formalism required for the interpretation of nonreciprocity is regrettably complex; in fact, all existing models of internal wave spectra give null results for the most interesting statistics (the cross correlation of reciprocal travel times). The first four sections prepare the way for a discussion of the previous experiment and of a future experiment for measuring momentum flux.
In Section 1 we examine the role of internal wave particle velocities in the scattering of sound; the contribution of velocity scattering is important for deep paths.

In Section 2 we deal with the representation of the internal wave field when the surface boundary condition of zero vertical velocity does not hold precisely, as would be the case for a vertical momentum flux transmitted through internal waves. A representation by wave packets in three-dimensional wavenumber space is convenient. The spectral model is generalized to provide for vertical asymmetry and horizontal anisotropy.

In Section 3 we derive the statistics for the acoustic phases $\phi_{ \pm}(t)$ of the pulsed transmissions in the $\pm x$-directions, as functions of the geophysical time $t$. (Phases are travel times multiplied by acoustic center frequency.) The mean products $\left\langle\phi_{+}{ }^{2}(t)\right\rangle,\left\langle\phi_{-}{ }^{2}(t)\right\rangle$, $\left\langle\phi_{+}(t) \phi_{-}(t)\right\rangle, \ldots$, can all be expressed as integrals of the internal wave spectra

$\int_{\text {ray }} d x \cdots \sum_{j} \cdots \int d \omega \cdots \int d \alpha \cdots S(j, \omega, \alpha ; z)$,

where $j, \omega, \alpha$ are the internal wave mode number, frequency and azimuth. The $\alpha$ integration is removed if the integrals are evaluated by stationary phase, and $\alpha=\alpha(\omega ; z)$. For a factored GM spectra $S(j, \omega)=H(j) G(\omega)$ the $\omega$ integration and $j$ summa- 
tion can be performed explicitly, and even the $x$ integration can be carried out for the special cases of an axial ray and of an upper loop (ray apex approximation).

A parallel derivation of the $\ln$-intensities, is given in Section 4 but with much more limited analytical results.

In Section 5 Worcester's experiment in reciprocal transmission in examined. The data set is severely deficient, and we regard this section as an illustration for evaluating the previous general expressions for a specific experimental situation. The solution of the forward problem depends, of course, on our assumptions concerning vertical asymmetry and horizontal anisotropy.

In Section 6 we examine the inverse problem of deriving useful information about internal waves without such ad hoc assumptions. The acoustically measured time series $\phi_{+}(t)$ and $\phi_{-}(t)$ provide us with two autospectra $P_{\phi_{+} \phi_{+}}(\omega)$ and $P_{\phi_{-} \phi_{-}}(\omega)$ and the co- and quadrature spectra, $P_{\phi_{+} \phi_{-}}(\omega)$ and $Q_{\phi_{+} \phi_{-}}(\omega)$. The acoustically measured spectra are related to the spectra and cross spectra of the vertical displacement $\zeta$ and the horizontal particle velocities $u, v$ of the internal wave field. The relation is through integral equations whose solution is a problem of inverse theory. Of particular interest is a relation between $Q_{\phi_{+} \phi_{-}}(\omega)$, the quadrature spectrum of reciprocal travel times, and the $\omega$-spectrum of $\left\langle u \partial_{t} \zeta\right\rangle$, i.e., the frequency spectrum of the vertical flux of horizontal momentum. It would be of great interest to measure the evolution of momentum flux as a function of frequency, depth and time during a storm episode.

Whether stable estimates of this kind can be made we do not know; the inversion problem is intricate at best and we have not attempted to solve it here. We shall try to gain some experimental experience over the next few years.

\section{Propagation velocity}

Let $\zeta(x, y, z, t)$ designate the upward particle displacement due to internal wave motion, and

$$
u, v, w=\partial_{t} \zeta
$$

the three components of internal wave particle velocities. The perturbation in sound speed arising from the vertical displacement equals $\zeta$ times the vertical gradient in potential (actual minus adiabatic) sound speed, i.e.,

$$
\delta c=\zeta\left(\partial_{z} c\right)_{\text {potential }} .
$$

Thus

$$
\delta c \pm u
$$

is the internal wave perturbation in propagation velocity in the $\pm x$ direction, ignoring the small tilt of
TABLE 1. Representative values of internal wave parameters. ${ }^{1}$

\begin{tabular}{lcccccc}
\hline \hline & $\begin{array}{c}z \\
(\mathrm{~km})\end{array}$ & $\begin{array}{c}n \\
(\mathrm{cph})\end{array}$ & $\begin{array}{c}\mathrm{rms} \zeta \\
(\mathrm{m})\end{array}$ & $\begin{array}{c}\mathrm{rms} u \\
\left(\mathrm{~cm} \mathrm{~s}^{-1}\right)\end{array}$ & rms $\delta c / c$ & $\mathrm{rms} u / c$ \\
\hline Thermocline & 0 & 3 & 7.3 & 4.70 & $49 \times 10^{-5}$ & $3.1 \times 10^{-5}$ \\
Sound axis & -1 & 1.1 & 12.0 & 2.85 & $11 \times 10^{-5}$ & $1.9 \times 10^{-5}$ \\
& -2 & 0.406 & 19.8 & 1.73 & $2.4 \times 10^{-5}$ & $1.1 \times 10^{-5}$ \\
Bottom & -4.5 & 0.094 & 41.2 & 0.83 & $0.3 \times 10^{-5}$ & $0.6 \times 10^{-5}$ \\
\hline
\end{tabular}

sound rays. The fractional perturbation in propagation velocity is

$$
\mu_{ \pm}=\frac{\delta c}{c} \pm \frac{u}{c} \text {. }
$$

For a "canonical ocean" (Munk, 1974), Eq. (2) can be written

$$
\frac{\delta c}{c}=24.5 g^{-1} n^{2}(z) \zeta
$$

where

$$
n=n_{0} e^{z / b}
$$

is the Brunt-Väisälä (or buoyancy) frequency, $n_{0}$ the surface-extrapolated value, $b$ the scale depth, $g$ gravity ( $z$ is positive upwards). Constancy of internal wave vertical energy flux (the WKBJ approximation) requires that

$$
\operatorname{rms} \zeta \approx n^{-1 / 2}, \quad \operatorname{rms} u \approx n^{1 / 2}
$$

hence

$$
\text { rms } \delta c / c \sim n^{3 / 2} .
$$

Table 1 gives representative numerical values for the special case of a sound axis at $z=-b=-1 \mathrm{~km}$. Thus $\delta c$ and $u$ contributions from internal waves are comparable beneath $2 \mathrm{~km}$.

\section{Internal wave spectra}

The customary representation of an internal wave field is a linear superposition of elementary wave trains of random phase. The usual boundary condition has vanishing vertical velocity at the surface ${ }^{2}$ and on the bottom, and therefore a representation in terms of normal modes is more convenient. One writes the vertical displacement $\zeta(\mathbf{x}, t)$ as

$$
\begin{aligned}
& \zeta(\mathbf{x}, t) \\
& =\sum_{j} \int \frac{d^{2} \mathbf{k}_{H}}{(2 \pi)^{2}}\left\{a_{j}\left(\mathbf{k}_{H}\right) \exp \left[+i\left(\mathbf{k}_{H} \cdot \mathbf{x}_{H}-\omega t\right)\right] W_{j}\left(\mathbf{k}_{H} ; z\right)\right. \\
& \left.\quad+a_{j}^{*}\left(\mathbf{k}_{H}\right) \exp \left[-i\left(\mathbf{k}_{H} \cdot \mathbf{x}_{H}-\omega t\right)\right] W_{j}\left(\mathbf{k}_{H} ; z\right)\right\}, \quad(7)
\end{aligned}
$$

${ }^{1}$ From W. H. Munk and F. Zachariasen (1976), to be referred to as MZ. Table 1 of MZ is in error with regard to $u / c$. For a systematic treatment we refer to Flatté et al. (1979).

"The "free surface" boundary condition is replaced by the "lid" boundary; this approximation is adequate for the purpose of the present discussion. 
where $\omega=\omega_{j}\left(\mathbf{k}_{H}\right)$ is the frequency, $\mathbf{k}_{H}$ the horizontal wavenumber and $\mathbf{x}_{H}$ is the horizontal position. The mode functions $W_{j}\left(\mathbf{k}_{H} ; z\right)$ vanish at the surface $(z$ $=0)$ and at the bottom $(z=-D)$, so that the boundary condition is automatically built in.

If desired, one can equally well describe $\zeta$ in terms of plane waves with vertical as well as horizontal components of wavenumbers:

$$
\begin{aligned}
\zeta(\mathbf{x}, t)=\int d^{3} \mathbf{k} /(2 \pi)^{3}\{a(\mathbf{k}) \exp [+i(\mathbf{k} \cdot \mathbf{x}-\omega t) \\
\left.+a^{*}(\mathbf{k}) \exp [-i(\mathbf{k} \cdot \mathbf{x}-\omega t)]\right\} .
\end{aligned}
$$

Here $\mathbf{k}=\left(\mathbf{k}_{H}, k_{z}\right)$ and $\mathbf{x}=\left(\mathbf{x}_{H}, z\right)$. The (approximate) dispersion relation is

$$
k_{z}= \pm\left|\mathbf{k}_{H}\right|\left(\frac{n^{2}-\omega^{2}}{\omega^{2}-\omega_{\text {in }}^{2}}\right)^{1 / 2} .
$$

This simply expresses the well-known fact that the inclination $\tan \gamma=k_{z} /\left|\mathbf{k}_{H}\right|$ of the wavenumber to the horizontal is a function only of frequency.

But the boundary condition of zero vertical velocity at the surface does not hold precisely, as there is some (possibly very little) net vertical momentum flux transmitted through internal waves. When this flux is an essential element of the analysis, as it is here, (7) is incorrect but the more general representation (8) can still be used.

In the representation (8), positive (negative) values of $k_{z}$ represent waves having upward (downward) phase propagation, and downward (upward) energy propagation.

Thus if $a(\mathbf{k})$ is an even function of $k_{z}$, we have vertical symmetry. Conversely, if $a(\mathbf{k})$ has a component odd in $k_{z}$, (8) can represent asymmetric vertical propagation. Eq. (7), in contrast, is intrinsically vertically symmetric.

Müller et al. (1978) have made a consistency analysis of $1444 \zeta, u, v$ cross spectra collected during the IWEX experiment (Briscoe, 1975). They find that (i) the observed fluctuations are consistent with the random superposition of linear internal waves; (ii) the internal wave field is nearly horizontally isotropic and vertically symmetric except at inertial and tidal frequencies; (iii) WKBJ-scaling is satisfactory except at the turning points where more refined methods (e.g., the Airy solution) should be used; and (iv) at intermediary frequencies, normal modes and plane waves are about equally satisfactory. At buoyant frequencies $(\omega \rightarrow n)$ the plane wave representation is inconvenient to describe the measurements (presumably because the upward and downward propagating waves have increasingly deterministic phase relations with proximity to the turning point). To these findings we can add the expectation that $(v)$ the plane wave representation is inconveni- ent to describe the largest vertical wavelengths (low modes) for reasons similar to those stated in (iv).

Other empirical evidence also favors isotropy and symmetry over the opposite extreme of plane waves in a narrow beam (for a review, see Garrett and Munk, 1972, 1975) and this view has received support from recent theoretical work (McComas, 1977; Pomphrey et al., 1980) which shows that a narrow beam is rapidly diffused (equipartioned) as a result of nonlinear interactions. Certainly the assumptions of isotropy and symmetry greatly simplify the problem. But here we cannot take advantage of this simplification, because the nonreciprocity of acoustic transmissions depends crucially on the covariance $\langle\zeta u\rangle$ which, as will be seen, vanishes in the case of either isotropy or symmetry (or both). At the same time there is much to be gained from studying these departures from isotropy and symmetry even if they are slight, for these departures are closely related to the vertical momentum flux $\langle w u\rangle$ in an internal wave field.

The random phase assumption asserts that statistical averages of the wave amplitudes are uncorrelated. That is,

$$
\left\langle a(\mathbf{k}) a\left(\mathbf{k}^{\prime}\right)\right\rangle=\left\langle a^{*}(\mathbf{k}) a^{*}\left(\mathbf{k}^{\prime}\right)\right\rangle=\mathbf{0},
$$

while

$$
\left\langle a(\mathbf{k}) a^{*}\left(\mathbf{k}^{\prime}\right)\right\rangle=1 / 2(2 \pi)^{3} \delta^{3}\left(\mathbf{k}-\mathbf{k}^{\prime}\right) S(\mathbf{k}) .
$$

Since in general we are allowing both even and odd (in $k_{z}$ ) parts of $a$, there will be even and odd (in $k_{z}$ ) parts of $S$; these we denote ${ }^{3}$ by $S^{(+)}$and $S^{(-)}$:

$$
S(\mathbf{k})=S^{(+)}\left(\mathbf{k}_{H}, k_{z}\right)+S^{(-)}\left(\mathbf{k}_{H}, k_{z}\right),
$$

where

$$
S^{( \pm)}\left(\mathbf{k}_{H}, k_{z}\right)= \pm S^{( \pm)}\left(\mathbf{k}_{H},-k_{z}\right) .
$$

The spectrum $S$ is related to the mean-square displacement according to (10) and (11):

$$
\left\langle\zeta^{2}\right\rangle=\left\{\begin{array}{l}
\int \frac{d^{3} \mathbf{k}}{(2 \pi)^{3}} S(\mathbf{k}) \\
2 \int_{0}^{\infty} \frac{d k_{z}}{2 \pi} \int \frac{d^{2} \mathbf{k}_{H}}{(2 \pi)^{2}} S^{(+)}\left(\mathbf{k}_{H}, k_{z}\right),
\end{array}\right.
$$

since $\int_{-\infty}^{\infty} d k_{z} S^{(-)}=0$. We can, if we wish, re-express this in terms of mode number $j$ (defined through the approximate relation

$$
k_{H}=j \pi\left(\omega^{2}-\omega_{i n}^{2}\right)^{1 / 2} / n_{0} b
$$

as given in GM72), frequency $\omega$, defined in (9), and horizontal azimuth $\alpha$, so that

$$
k_{x}=k_{H} \cos \alpha, \quad k_{y}=k_{H} \sin \alpha, k_{z}=k_{H} \tan \gamma .
$$

\footnotetext{
${ }^{3}$ The superscripts $( \pm)$ are of course unrelated to the subscripts \pm in $\mathrm{Eq}$. (4) which designate propagation in the $\pm x$ direction.
} 
Thus we write

$$
\left\langle\zeta^{2}\right\rangle=\sum_{j} \int_{\omega_{i n}}^{n} d \omega \int_{0}^{2 \pi} \frac{d \alpha}{2 \pi} S(\omega, j, \alpha),
$$

with a relation between the spectrum $S(\omega, j, \alpha)$ and the spectra $S^{(+)}(\mathbf{k})$.

The velocity components $u, v$ and $w$ can be obtained from the vertical displacement $\zeta$ by using the equations of motion. We have

$$
\begin{aligned}
u(\mathbf{x}, t)=\int \frac{d^{3} \mathbf{k}}{(2 \pi)^{3}}\left\{\tan \gamma\left(i \omega \cos \alpha-\omega_{i n} \sin \alpha\right)\right. \\
\quad \times \exp [+i(\mathbf{k} \cdot \mathbf{x}-\omega t)] a(\mathbf{k}) \\
+\tan \gamma\left(-i \omega \cos \alpha-\omega_{i n} \sin \alpha\right) \\
\left.\quad \quad \quad \quad \exp [-i(\mathbf{k} \cdot \mathbf{x}-\omega t)] a^{*}(\mathbf{k})\right\},
\end{aligned}
$$

with a similar expression for $v$. The vertical velocity $w=\dot{\zeta}$ is

$$
\begin{array}{r}
w(\mathbf{x}, t)=\int \frac{d^{3} \mathbf{k}}{(2 \pi)^{3}}\{-i \omega \exp [+i(\mathbf{k} \cdot \mathbf{x}-\omega t)] a(\mathbf{k}) \\
\left.+i \omega \exp [-i(\mathbf{k} \cdot \mathbf{x}-\omega t)] a^{*}(\mathbf{k})\right\}
\end{array}
$$

From these we deduce, in analogy to (14), that

$$
\begin{aligned}
\left\langle u^{2}\right\rangle= & \int \frac{d^{3} \mathbf{k}}{(2 \pi)^{3}} \\
& \times \tan ^{2} \gamma\left(\omega^{2} \cos ^{2} \alpha+\omega_{i n} \sin ^{2} \alpha\right) S(\mathbf{k}), \\
\left\langle w^{2}\right\rangle= & \int \frac{d^{3} \mathbf{k}}{(2 \pi)^{3}} \omega^{2} S(\mathbf{k}) .
\end{aligned}
$$

These, again, may be written in $\omega, j, \alpha$ language. As in the case of $\left\langle\zeta^{2}\right\rangle$, only the even (in $k_{z}$ ) part of the spectrum $S(\mathbf{k})$ contributes, the coefficient in (19) and (20) being even functions of $k_{z}$.

The corresponding expressions for the covariances are

$$
\left.\begin{array}{rl}
\langle\zeta u\rangle & =-\int \frac{d^{3} \mathbf{k}}{(2 \pi)^{3}} \omega_{i n} \sin \alpha \tan \gamma S(\mathbf{k}) \\
\langle w u\rangle & =-\int \frac{d^{3} \mathbf{k}}{(2 \pi)^{3}} \omega^{2} \cos \alpha \tan \gamma S(\mathbf{k})
\end{array}\right\}
$$

Here, since $\tan \gamma$ is odd in $k_{z}$, only the odd part of $S$, namely $S^{(-)}$, will contribute.

To evaluate the expressions for the variances and covariances, we will use a model for the spectrum. In the usual model idealizations one assumes i) vertical symmetry, $S^{(-)}(\omega, j, \alpha)=0$, ii) horizontal isotropy, $S^{(+)}(\omega, j, \alpha)$ independent of $(\alpha)$, and these two assumptions are generally supported by the analysis of Müller et al. (1978), as previously mentioned. The covariances (21) vanish for either (or both) con- ditions; for i) because $S=S^{(-)}$in (21), and for ii) due to the integration over $\alpha$.

We shall use a generalization of the GM model (Garrett and Munk, 1972, 1975; Munk, 1980); for the even spectrum we arbitrarily assume that the angular dependence can be factored

with

$$
S_{\zeta \zeta}^{(+)}(\omega, j, \alpha)=H(j) E(\omega) F(\alpha),
$$

$$
\sum_{j=1}^{\infty} H(j)=1, \quad \int_{0}^{2 \pi} \frac{d \alpha}{2 \pi} F(\alpha)=1 .
$$

The GM mode weighting is

$$
H(j)=\frac{\left(j^{2}+j_{*}^{2}\right)^{-1}}{\sum_{j=1}^{\infty}\left(j^{2}+j_{*}^{2}\right)^{-1}}, j_{*}=3,
$$

and the frequency weighting function is

$E(\omega)=(4 / \pi) \omega_{i n}\left(\omega^{2}-\omega_{i n}^{2}\right)^{1 / 2} \omega^{-3}\left\langle\zeta^{2}\right\rangle$

with

$$
\equiv G_{\zeta \zeta}(\omega)\left\langle\zeta^{2}\right\rangle,
$$

$$
\int_{0}^{\infty} G_{\zeta \zeta}(\omega) d \omega=1, \quad\left\langle\zeta^{2}\right\rangle=\left(n_{0} / n\right)\left\langle\zeta_{0}^{2}\right\rangle
$$

and $\left\langle\zeta_{0}{ }^{2}\right\rangle^{1 / 2}=7.3 \mathrm{~m}$. The normalization is consistent with (16). We leave $F(\alpha)$ unspecified.

Nothing is known about the odd spectrum $S^{(-)}$ other than that it is small. For simplicity, we set

$$
S^{(-)}=r S^{(+)} \operatorname{sgn}\left(k_{z}\right)
$$

for some constant $r$; positive $r$ designates a net upward phase and downward energy propagation. Hereafter we drop the superscripts and write $S_{\zeta \zeta}$ $=S_{\zeta 5}^{(+)}$.

\section{Mean-square acoustic phase}

We first refer to the previous results ${ }^{1}$ for the effects of vertical particle displacements in an isotropic symmetric internal wave field. From MZ(115) and $M Z(118)$ we have $\left\langle\phi^{2}\right\rangle=\left\langle|X|^{2}\right\rangle$. Then from (MZ66)

$$
\begin{aligned}
\langle\phi \phi\rangle & =\frac{2}{\pi} q^{2} b \frac{n_{0}}{\omega_{i n}} \int_{0}^{R} d x \sec ^{2} \theta \\
& \times \sum_{j} j^{-1} \int_{\omega_{L}}^{n} d \omega \frac{\omega_{i n}}{\left(\omega^{2}-\omega_{L}^{2}\right)^{1 / 2}} S(\omega, j ; z),
\end{aligned}
$$

where $q$ is the acoustic wavenumber, $\omega_{L}{ }^{2}=\omega_{i n}{ }^{2}$ $+n^{2} \tan ^{2} \theta, \omega_{i n}$ is the inertial frequency, and $\theta$ the ray inclination relative to the horizontal. The integral $\int_{0}^{R} d x$ is along the ray from source to receiver. Further

$$
\sum_{j} \int_{\omega_{i n}}^{n} d \omega S(\omega, j)=\left\langle(\delta c / c)^{2}\right\rangle
$$


is the definition of the power spectrum $S(\omega, j)$ of $\delta c / c$ as a function of frequency $\omega$ and vertical modenumber $j$. Eq. (27) was derived for a horizontally isotropic and vertically symmetric spectrum. For an anisotropic medium, where $S$ depends on $\alpha$, Eq. (27) is modified by replacing $S(\omega, j ; z)$ by $S\left(\omega, j, \alpha_{0} ; z\right)$, with

$$
\tan \alpha_{0}=-\left(\omega^{2}-\omega_{L}^{2}\right)^{1 / 2} /(n \tan \theta) .
$$

The only values of $\alpha$ that contribute to the integral are $\alpha=\alpha_{0}$. The reason for this is that only those components of the internal wavenumber vector $k$ which are perpendicular to the acoustic ray and thereby of stationary internal wave phase contribute to acoustic phase fluctuations [for a correlation length of sound speed fluctuations small compared to the range $R$, as assumed in the derivation of (27)]. ${ }^{1}$ This geometric restraint, together with the internal wave relation (9), leads to

$$
\omega^{2}=\omega_{i n}^{2}+n^{2} \tan ^{2} \theta\left(1+\tan ^{2} \alpha\right)
$$

with a lower frequency limit given by $\omega_{L}^{2}=\omega_{i n}^{2}$ $+n^{2} \tan ^{2} \theta$, and the expression (29) for $\alpha_{0}$. Thus, along the ray, only two of the internal wave degrees of freedom are independent, and the value of the azimuth in (27) is determined by $\omega$ and $j$ [actually only by $\omega$, as we see from (29)].

To take the particle velocities into account, we need to replace $\left\langle(\delta c / c)^{2}\right\rangle$ by $\left\langle\mu^{2}\right\rangle(\mathrm{Eq} .4)$. This reproduces the foregoing results when $u=0$. When $u \neq 0$ however, we need to distinguish between the phase fluctuation $\phi_{+}(t)$ for a transmission in the positive $x$ direction, and $\phi_{-}(t)$ for a negative transmission. These depend on $\mu_{ \pm}(t)$ along the ray path. Eqs. (27) and (28) generalize to

$$
\begin{array}{r}
\left\langle\phi_{+} \phi_{+}\right\rangle=\cdots \int d x \\
\cdots \sum_{j} \cdots \int d \omega \cdots S_{\mu_{+} \mu_{+}} \\
\times\left\{\omega, j, \alpha_{0}[\omega, z(x)] ; z(x)\right\}
\end{array}
$$

and similarly for $\left\langle\phi_{-} \phi_{-}\right\rangle,\left\langle\phi_{+} \phi_{-}\right\rangle$, where

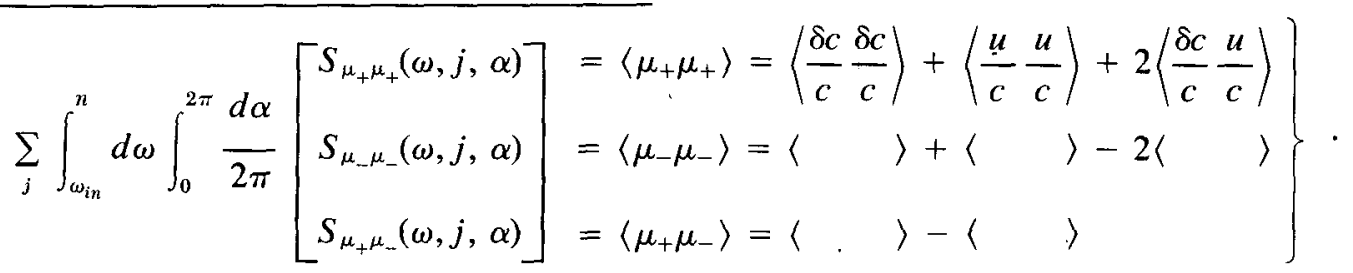

We now define the spectra ${ }^{4}$

$$
\begin{aligned}
& \sum_{i} \int_{\omega_{i n}}^{n} d \omega \int_{0}^{2 \pi} \frac{d \alpha}{2 \pi} S_{c c}, S_{u u}, S_{c u} \\
&=\left\langle\frac{\delta c}{c} \frac{\delta c}{c}\right\rangle,\left\langle\frac{u}{c} \frac{u}{c}\right\rangle,\left\langle\frac{\delta c}{c} \frac{u}{c}\right\rangle
\end{aligned}
$$

associated with vertical particle displacement, horizontal particle velocity and the cross term, so that

$$
S_{\mu_{+} \mu_{+}}=S_{c c}+S_{u u}+2 S_{c u}
$$

and similarly for $S_{\mu_{-} \mu_{-}}$and $S_{\mu_{+} \mu_{-}}$, using the sign convention in (31). $S_{c u}$ is the co-spectrum of $\delta c / c$ and $u / c$.

Combining (30), (31) and (32) leads to the rules

$$
\left.\begin{array}{l}
\left\langle\phi_{+} \phi_{+}\right\rangle=\Phi_{c c}+\Phi_{u u}+2 \Phi_{c u} \\
\left\langle\phi_{-} \phi_{-}\right\rangle=\Phi_{c c}+\Phi_{u u}-2 \Phi_{c u} \\
\left\langle\phi_{+} \phi_{-}\right\rangle=\Phi_{c c}-\Phi_{u u}
\end{array}\right\},
$$

where

$$
\begin{aligned}
\Phi_{c c} & =\frac{2}{\pi} q^{2} b \frac{n_{0}}{\omega_{i n}} \int_{0}^{R} d x \sec ^{2} \theta \sum_{j} \frac{1}{j} \int_{\omega_{L}}^{n} d \omega \\
& \times \frac{\omega_{i n}}{\left(\omega^{2}-\omega_{L}^{2}\right)^{1 / 2}} S_{c c}\left\{\omega, j, \alpha_{0}[\omega, z(x)] ; z(x)\right\}
\end{aligned}
$$

${ }^{4}$ In the subscripts (but not otherwise) we use the abbreviation $c$ for $\delta c / c$ and $u$ for $u / c$. and similarly for $\Phi_{u u}, \Phi_{c u}$ with $S_{u u}, S_{c u}$. Since $\left\langle\zeta^{2}\right\rangle$ is proportional to $\left\langle(\delta c / c)^{2}\right\rangle$,

$$
S_{c c}(\omega, j, \alpha)=\kappa_{c}^{2}\left(n / n_{0}\right)^{4} S_{\zeta \zeta}(\omega, j, \alpha),
$$

where

$$
\kappa_{c}=24.5 n_{0}{ }^{2} / g=6.8 \times 10^{-5} \mathrm{~m}^{-1} .
$$

Further, from Eq. (19) we have

$S_{u u}(\omega, j, \alpha)=\kappa_{u}^{2} n_{0}^{-2}\left(\omega^{2} \cos ^{2} \alpha+\omega_{i n}^{2} \sin ^{2} \alpha\right)$

and from Eq. (21)

$$
\times \tan ^{2} \gamma S_{\zeta \zeta}(\omega, j, \alpha),
$$

$S_{c u}(\omega, j, \alpha)=\kappa_{u} \kappa_{c} n_{0}^{-1}\left(n / n_{0}\right)^{2} \omega_{i n} \sin \alpha$

where

$$
\times \tan \gamma r S_{\zeta \zeta}(\omega, j, \alpha),
$$

$$
\kappa_{u}=n_{0} / c=3.5 \times 10^{-6} \mathrm{~m}^{-1} .
$$

In the above expressions, $\alpha$ is to be evaluated at $\alpha_{0}[\omega, z(x)]$ when integrating along the ray. From Eq. (29) we have

$$
\left.\begin{array}{l}
\cos \alpha_{0}=\frac{n \tan \theta}{\left(\omega^{2}-\omega_{i n}^{2}\right)^{1 / 2}} \\
\sin \alpha_{0}=-\frac{\left(\omega^{2}-\omega_{L}^{2}\right)^{1 / 2}}{\left(\omega^{2}-\omega_{i n}^{2}\right)^{1 / 2}}
\end{array}\right\},
$$

and can rewrite (37) and (38) in the form 


$$
\begin{aligned}
& S_{u u}\left(\omega, j, \alpha_{0}\right) \\
& =n_{0}{ }^{-2} \kappa_{u}{ }^{2} \omega_{L}{ }^{2}\left(\frac{n^{2}-\omega^{2}}{\omega^{2}-\omega_{i n}^{2}}\right) S_{\zeta \zeta}\left(\omega, j, \alpha_{0}\right), \\
& S_{c u}\left(\omega, j, \alpha_{0}\right)=-n_{0}{ }^{-1} \kappa_{u} \kappa_{c}\left(\frac{n}{n_{0}}\right)^{2} \\
& \times \frac{\omega_{i n}\left(\omega^{2}-\omega_{L}^{2}\right)^{1 / 2}\left(n^{2}-\omega^{2}\right)^{1 / 2}}{\omega^{2}-\omega_{i n}^{2}} r S_{\zeta \zeta}\left(\omega, j, \alpha_{0}\right) .
\end{aligned}
$$

For the GM spectrum, $S_{\zeta \zeta}$ is proportional to $\left(\omega^{2}\right.$ - $\left.\omega_{i n}^{2}\right)^{1 / 2}$ and $S_{u u}\left(\alpha_{0}\right)$ and $S_{c u}\left(\alpha_{0}\right)$ are strongly weighted toward $\omega_{i n}$, whereas $S_{c c}$ vanishes at $\omega_{i n}$ (inertial motion is horizontally polarized). The principal contributions to nonreciprocity come from inertial frequencies.

Using the internal wave spectral model given in the preceding section,

$$
S_{\zeta \zeta}(\omega, j, \alpha)=H(j) G_{\zeta \zeta}(\omega) F(\alpha)\left\langle\zeta^{2}\right\rangle,
$$

it is now possible to evaluate $\Phi_{c c}, \Phi_{u u}$, and $\Phi_{c u}$ for various choices of the angular distribution $F(\alpha)$. Since the angular dependence of the spectrum is in fact weak, we will assume horizontal isotropy, $F(\alpha)=1$. Only the dependence on vertical asymmetry then remains. Evaluating Eq. (34) then gives ${ }^{5}$

$$
\begin{aligned}
& \left\{\begin{array}{c}
\Phi_{c c} \\
\Phi_{u u} \\
\Phi_{c u}
\end{array}\right\}=2 \pi^{-1} q^{2} b \frac{n_{0}}{\omega_{i n}}\left\langle j^{-1}\right\rangle \\
& \quad \times\left\{\begin{array}{c}
\int d x \sec ^{2} \theta \kappa_{c}{ }^{2}\left(\frac{n}{n_{0}}\right)^{3}\left\langle\zeta_{0}{ }^{2}\right\rangle \Omega_{c c} \\
\int d x \sec ^{2} \theta \kappa_{u}{ }^{2}\left(\frac{n}{n_{0}}\right)\left\langle\zeta_{0}{ }^{2}\right\rangle \Omega_{u u} \\
r \int d x \sec ^{2} \theta \kappa_{c} \kappa_{u}\left(\frac{n}{n_{0}}\right)^{2}\left\langle\zeta_{0}{ }^{2}\right\rangle \Omega_{c u}
\end{array}\right\},
\end{aligned}
$$

where

$$
\begin{aligned}
& \Omega_{c c}=\frac{2}{\pi}\left(\frac{1}{D^{2}}+\frac{D^{2}-1}{2 D^{3}} \ln \frac{D+1}{D-1}\right), \\
& \Omega_{u u}=\frac{1}{\pi}\left(-2+\frac{D^{2}+1}{D} \ln \frac{D+1}{D-1}\right), \\
& \Omega_{c u}=-\frac{1}{D^{3}},
\end{aligned}
$$

${ }^{5}$ I. S. Gradshteyn and I. M. Ryzhik, Table of Integrals, Series and Products (Academic Press, 1965). Formula 2.264 is helpful For $\Omega_{c c}$ use the integration variable $x=1-\omega_{i n}^{2} / \omega^{2}$ and multiply numerator and denominator $\sqrt{x}$; for $\Omega_{u u}$ use $x=\omega_{i n}^{2} / \omega^{2}$. with

$$
D^{2}=\frac{\omega_{L}^{2}}{\omega_{i n}^{2}}=1+\frac{n^{2} \tan ^{2} \theta}{\omega_{i n}^{2}} .
$$

We also have

$$
\left\langle j^{-1}\right\rangle=\sum_{1}^{\infty} j^{-1} H(j)=0.44
$$

using our choice of $H(j)$. The integrands in (42) are given functions of $\theta(z)$ and $n(z)$ which vary along the ray paths $z(x)$. It is a simple matter to compute the ray paths from Snell's law, and to perform the integrations numerically. The mean-square phases follow at once from Eq. (33). But for two special cases the solution can be obtained analytically.

\section{a. Axial approximation}

For the case of a transmission along the sound axis, $z=z_{1}, D=1, \Omega_{c c}=2 / \pi$, and $\Omega_{c u}=-1$; however $\Omega_{u u}=\infty$ and (44) requires an asymptotic expansion near the axis (Appendix A). The result is

$$
\begin{aligned}
& \left\{\begin{array}{c}
\Phi_{c c} \\
\Phi_{u u} \\
\Phi_{c u}
\end{array}\right\}=2 \pi^{-1} q^{2} b R \frac{n_{0}}{\omega_{i n}}\left\langle j^{-1}\right\rangle \\
& \times\left\{\begin{array}{c}
\frac{2}{\pi} \kappa_{c}{ }^{2}\left(\frac{n_{1}}{n_{0}}\right)^{3}\left\langle\zeta_{0}{ }^{2}\right\rangle \\
\kappa_{u}{ }^{2}\left(\frac{n_{1}}{n_{0}}\right)\left\langle\zeta_{0}{ }^{2}\right\rangle\left(\frac{4}{\pi} \ln \frac{4 R_{0}}{9.2\left|R-R_{0}\right|}\right) \\
-r \kappa_{c} \kappa_{u}\left(\frac{n_{1}}{n_{0}}\right)^{2}\left\langle\zeta_{0}{ }^{2}\right\rangle
\end{array}\right\},
\end{aligned}
$$

where $n_{1}=n\left(z_{1}\right)$. Here $R_{0}=21 \mathrm{~km}$ is the range of a canonical axial loop. The three terms in the righthand brackets for the sound axis at $z_{1}=-b$, hence $n_{1} / n_{0}=e^{-1}$ are

$$
7.8 \times 10^{-9}, \quad \ln \left(0.43 R_{0} /\left|R-R_{0}\right|\right) \times 2.4 \times 10^{-10},
$$

$$
-1.7 \times 10^{-9} r \text {. }
$$

The middle term dominates when $R$ is very close to $R_{0}$, but even for $R=1.01 R_{0}$ it is $9 \times 10^{-10}$ and so small as compared to the first term. In the case of vertical symmetry $(r=0),\left\langle\phi_{+} \phi_{+}\right\rangle,\left\langle\phi_{-} \phi_{-}\right\rangle$and $\left\langle\phi_{+} \phi_{-}\right\rangle$are then nearly the same.

\section{b. Apex approximation}

For steep rays the major contribution to the $x$ integrals come from the upper apex of the ray. Near an apex located at $(\hat{x}, \hat{z})$, the equation of the ray is $z(x)=\hat{z}-(x-\hat{x})^{2} / 2 \hat{\mathscr{R}}$ where $\hat{\mathscr{R}}$ is the ray curvature at the turning point. From MZ85 we have for the canonical sound channel that 


$$
\hat{R}^{-1}=\frac{2 \epsilon}{b_{c}} \frac{c_{1}}{\hat{c}}|1-\exp \hat{\eta}| \approx \gamma_{A}|1-\exp \hat{\eta}|
$$

where $\left.c(z)=c_{1}\{1+\epsilon[\exp (\eta)-\eta-1]\}, \quad \eta\right)=(z$ $\left.-z_{1}\right) / 1 / 2 b_{c}, b_{c}$ is the sound-speed scale depth, $z_{1}$ is the axis depth, and $\gamma_{A}=1.1 \times 10^{-2} \mathrm{~km}^{-1}$. Further

$$
D^{2}=1+\frac{n^{2}}{\omega_{i n}^{2}} \tan ^{2} \theta \approx 1+\left(\frac{\hat{n}}{\omega_{i n}} \frac{x-\hat{x}}{\hat{\Re}}\right)^{2} .
$$

In performing the integrations in (42) we set $\theta=0$, $n=\hat{n}$, and write $d x=\hat{R}\left(\omega_{i n} / \hat{n}\right) D\left(D^{2}-1\right)^{-1 / 2} d D$ from (49). The result is

$$
\begin{aligned}
\left\{\begin{array}{l}
\Phi_{c c} \\
\Phi_{u u} \\
\Phi_{c u}
\end{array}\right\}=2 q^{2} b \hat{\mathscr{R}}\left\langle j^{-1}\right\rangle \frac{n_{0}}{\hat{n}} \\
\times\left\{\begin{array}{c}
\kappa_{c}{ }^{2}\left(\frac{\hat{n}}{n_{0}}\right)^{3}\left\langle\zeta_{0}{ }^{2}\right\rangle \\
0.48 \kappa_{u}{ }^{2}\left(\frac{\hat{n}}{n_{0}}\right)\left\langle\zeta_{0}{ }^{2}\right\rangle \\
-r \frac{2}{\pi} \kappa_{c} \kappa_{u}\left(\frac{\hat{n}}{n_{0}}\right)^{2}\left\langle\zeta_{0}{ }^{2}\right\rangle
\end{array}\right\}
\end{aligned}
$$

from each turning point. For a turning point near the surface we have $\hat{z} \approx 0$ and $\hat{n} \approx n_{0} . \Phi_{c c}, \Phi_{u u}, \Phi_{c u}$ are in the proportion $\{25,0.03,-0.81 r\}$, with the first term clearly dominating, so that $\left\langle\phi_{+} \phi_{+}\right\rangle$, $\left\langle\phi_{-} \phi_{-}\right\rangle$and $\left\langle\phi_{+} \phi_{-}\right\rangle$are again nearly equal.

\section{Mean-square intensity}

We define the zero-meaned intensity ${ }^{6}$

$$
\iota==i-\langle\bar{\imath}\rangle, \quad \tilde{\imath}=\ln p^{2}, \quad\langle i\rangle=\ln p_{0}{ }^{2},
$$

where $p(t)$ is the slowly varying amplitude of the received pressure oscillation $p e^{i \omega t}$ from the transmitted signal at frequency $\omega$, and $p_{0}$ is the amplitude in the absence of ocean variability. In analogy with the Eqs. (33) we write

$$
\left\langle\iota_{+} \iota_{+}\right\rangle=I_{c c}+I_{u u}+2 I_{c u}
$$

and similarly for $\left\langle\iota_{-} \iota_{-}\right\rangle,\left\langle\iota_{+} \iota_{-}\right\rangle$. Generalizing $M Z(116)$ to allow anisotropy gives ${ }^{7}$

$$
\begin{aligned}
& I_{c c}=\operatorname{Re}\left\{\frac{4}{\pi} q^{2} b \frac{n_{0}}{\omega_{i n}}\right. \\
& \quad \times \int_{0}^{R} d x \sec ^{2} \theta \sum_{j} j^{-1}\left(1-e^{i \beta j z}\right) \\
& \quad \times \int_{\omega_{L}}^{n} d \omega \frac{\omega_{i n}}{\left(\omega^{2}-\omega_{L}^{2}\right)^{1 / 2}} \\
& \text { with } \left.\quad \times S_{c c}\left\{\omega, j, \alpha_{0}[\omega, z(x)] ; z(x)\right\}\right\}, \\
& \quad \beta=(\pi / b)^{2}\left(n / n_{0}\right)^{2}(1 / q A) .
\end{aligned}
$$

$A$ is the "phase curvature" of the ray which can be evaluated numerically.$^{8}$ For a ray consisting of a single downward loop, we may to a good approximation take

$$
A^{-1}=x(R-x) / R \quad \text { (downward loop). }
$$

We recognize that the frequency integrals in (53) are of the same form as for the phases in (34). Proceeding as in the previous section, we evaluate (53) using the spectral model given in Section (2) for the special case of horizontal isotropy.

The ranges and acoustic wavenumbers here under consideration are such that $\beta \ll 1$. In this event we may approximate the $j$ summation (see MZ120); the result is

$$
\left\{\begin{array}{c}
I_{c c} \\
I_{u u} \\
I_{r u}
\end{array}\right\}=2 \pi^{2} \frac{q}{b} \frac{n_{0}}{\omega_{i n}} \frac{j_{*}{ }^{2}}{\pi j_{*}{ }^{-1}}\left\{\begin{array}{c}
\int d x \sec ^{2} \theta \kappa_{c}{ }^{2}\left(\frac{n}{n_{0}}\right)^{5}\left\langle\zeta_{0}{ }^{2}\right\rangle\left|A^{-1}(x)\right| \Omega_{c c}(\theta, n) \\
\int d x \sec ^{2} \theta \kappa_{u}{ }^{2}\left(\frac{n}{n_{0}}\right)^{3}\left\langle\zeta_{0}{ }^{2}\right\rangle\left|A^{-1}(x)\right| \Omega_{u u}(\theta, n) \\
r \int d x \sec ^{2} \theta \kappa_{c} \kappa_{u}\left(\frac{n}{n_{0}}\right)^{4}\left\langle\zeta_{0}{ }^{2}\right\rangle\left|A^{-1}(x)\right| \Omega_{c u}(\theta, n)
\end{array}\right\} .
$$

It is not useful to attempt either an axial or an apex approximation. The phase curvature is not well approximated by (55) for either an upward loop or an axial ray, and for a downward loop the contribution at the apex does not dominate. Therefore we shall leave (56) as it stands, and be content with a numerical evaluation of the integrals.

${ }^{{ }^{i j}}$ Multiply by $10 / \ln 10$ to get the intensities in decibels.

\section{An experiment in reciprocal transmission}

The only mid-ocean experiment in simultaneous two-way transmissions known to us was conducted

\footnotetext{
' Flatté et al. (1980) have revised the predictions of MZ for the frequency spectrum of intensity fluctuations to include the effect of the vertical advection of short vertical wavelength internal waves by long vertical wavelength internal waves. The total variance is unchanged.

${ }^{*}$ See Eq. (7.1.10) of Flatté et al. (1979).
} 


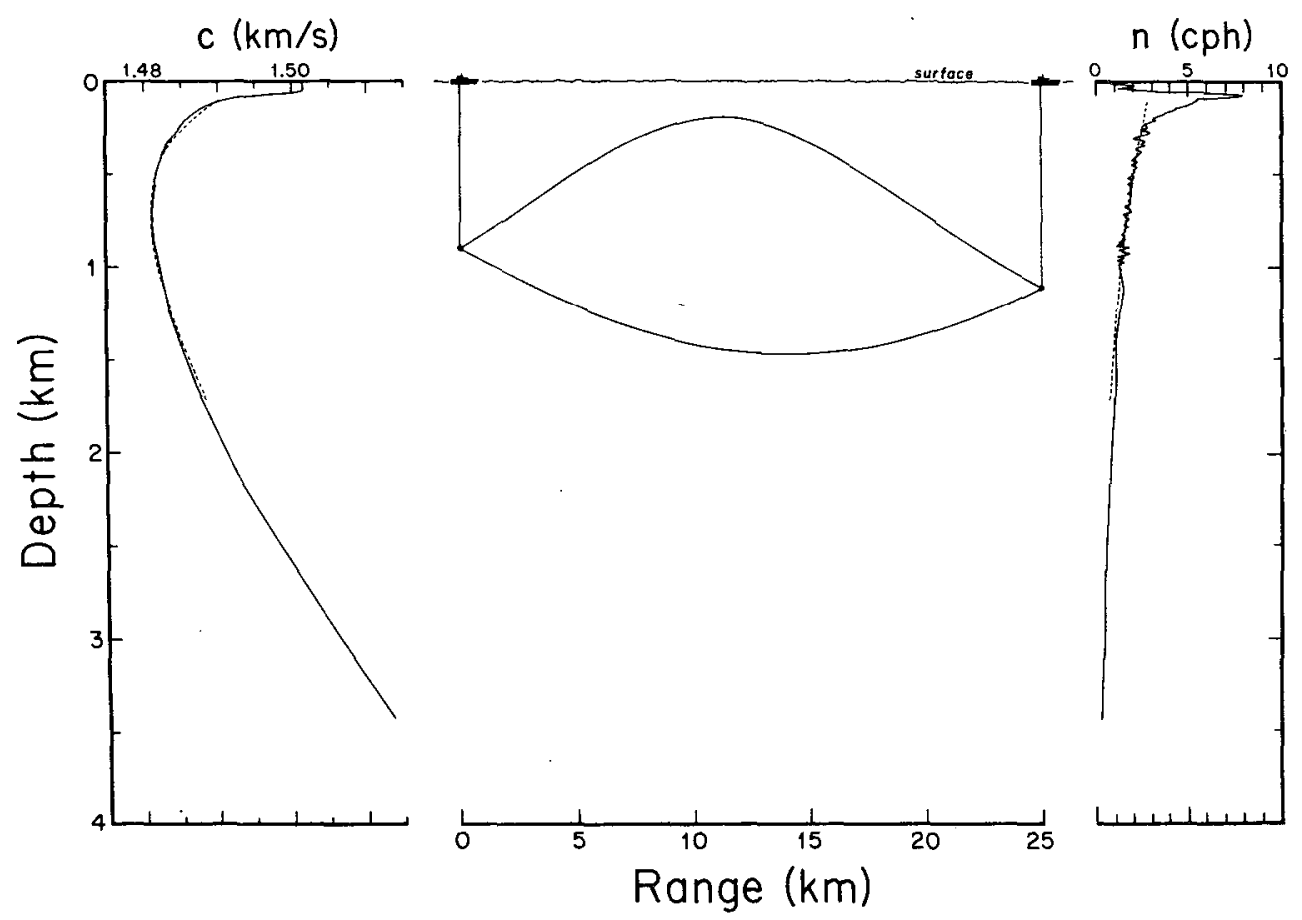

Fig. 1. Average sound-speed $(c)$ and buoyancy frequency $(n)$ profiles (solid lines). The associated ray geometry has two refracted paths with travel times differing by $40 \mathrm{~ms}$. Dashed lines give smooth fits to the profiles and are used in the theoretical computations (adapted from Worcester, 1977).

by Worcester $(1977,1979)$ approximately $350 \mathrm{~km}$ WSW of San Diego, California. Broadband acoustic transceivers centered at $2250 \mathrm{~Hz}$ were suspended from each of two ships at about $1 \mathrm{~km}$ depth and $25 \mathrm{~km}$ range (Fig. 1); this geometry was selected to give two purely refracted ray paths with travel times differing by $\sim 40 \mathrm{~ms}$. Phase-coded pulses were simultaneously transmitted every $30 \mathrm{~s}$ at each transceiver and were processed after reception to yield $\sim 0.6 \mathrm{~ms}$ time resolution.

The data set resulting from this experiment is severely deficient as an experimental test of the foregoing theory on several counts: (i) The available time series consists of only about $8 \mathrm{~h}$ of useful data out of a total of $15 \mathrm{~h}$, while most of the contribution to the current-related fluctuations is expected to come from near the inertial frequency of about

TABLE 2. Strength parameter $\Phi$ and diffraction parameter $\Lambda$ at $2250 \mathrm{~Hz}$ (adapted from Worcester, 1979).

\begin{tabular}{lcc}
\hline & Lower path & Upper path \\
\hline$\Phi(\mathrm{rad})$ & 15.0 & 30.4 \\
$\Lambda$ & $2.6 \times 10^{-3}$ & $4.6 \times 10^{-3}$ \\
$\Phi^{2} \Lambda$ & 0.6 & 4.3 \\
$\Phi \Lambda$ & 0.04 & 0.14 \\
Regime & Unsaturated & Partially saturated \\
& $\Phi^{2} \Lambda<1$ & $\Phi^{2} \Lambda>1, \Phi \Lambda<1$
\end{tabular}

one cycle per day; (ii) the principal source of phase fluctuation in the experiment was relative drift of the ships, making phase statistics useless; and (iii) at the center frequency of $2250 \mathrm{~Hz}$ used in the experiment, only the lower path is in the unsaturated regime for which the Rytov approximation used by Munk and Zachariasen (1976) is adequate (Table 2). Only the intensity fluctuations along the lower path are therefore available for comparison with theory.

We regard the experiment principally as a way of focusing on a specific situation for which the various predictions of the foregoing theory can be evalu-

TABLE 3. In-intensity covariances at $2250 \mathrm{~Hz}$ for reciprocal transmissions at $25 \mathrm{~km}$ range (multiply by $(10 / \mathrm{ln} 10)^{2}=18.86$ to get $\left.\mathrm{dB}^{2}\right)$. No theoretical value is available for $\left\langle\iota_{+} \iota_{-}\right\rangle$in the partially saturated regime. The positive direction is from the Alexander Agassiz to the Ellen B. Scripps.

\begin{tabular}{lccc}
\hline \hline & $\left\langle\iota_{+}\right\rangle$ & $\left\langle\iota_{+}\right\rangle$ & $\left\langle\iota_{-}\right\rangle$ \\
\hline Upper path & & & \\
$\quad$ Theoretical & 1.64 & 1.64 & $-\overline{\left.\iota_{-}\right\rangle}$ \\
Measured & 1.2 & 1.5 & 0.2 \\
& & & \\
Lower path & & & \\
$\quad$ Exact numerical & $0.79-0.22 r$ & $0.79+0.22 r$ & 0.72 \\
$\quad$ calculations & $0.68-0.18 r$ & $0.68+0.18 r$ & 0.62 \\
Approximation for $A^{-1}$ & 1.3 & 0.7 & 0.1 \\
Measured & & &
\end{tabular}


TABLE 4. Theoretical phase and In-intensity covariances for reciprocal transmissions at an acoustic frequency of $100 \mathrm{~Hz}$ and $25 \mathrm{~km}$ range. Upper and lower path values are for the geometry used by Worcester. Results for an axial path are included for comparison. Parameter values used include $q=419 \mathrm{~km}^{-1}, n_{0}=5.41 \times 10^{-3} \mathrm{~s}^{-1}(3.1 \mathrm{cph}), b=1.2 \mathrm{~km}, c_{1}=1.481 \mathrm{~km} \mathrm{~s}-1, \epsilon=0.003, b_{c}=0.8 \mathrm{~km}$, $z_{1}=-0.7 \mathrm{~km}, \omega_{i n}=7.3 \times 10^{-5} \mathrm{~s}^{-1}, j_{*}=3$, and $\left\langle j^{-1}\right\rangle=0.435$. For the upper path apex approximation, apex height $\hat{z}=-0.219 \mathrm{~km}$ and ray curvature $\hat{R}=57.5 \mathrm{~km}$ were used. Numerical calculations were done for two slightly different source angles, $\pm 0.1^{\circ}$, for the axial path. Phase covariances are in $(\mathrm{rad})^{2}$; multiply ln-intensity variances by $(10 / \mathrm{ln} 10)^{2}=18.86$ to obtain $(\mathrm{dB})^{2}$.

\begin{tabular}{|c|c|c|c|c|c|c|}
\hline & $\left\langle\phi_{+} \phi_{+}\right)$ & $\left\langle\phi_{-} \phi_{-}\right\rangle$ & $\left\langle\phi_{+} \phi_{-}\right\rangle$ & $\left\langle\iota_{+} \iota_{+}\right\rangle$ & $\left\langle\iota_{-} \iota_{-}\right\rangle$ & $\left\langle\imath_{+} \varepsilon_{-}\right\rangle$ \\
\hline \multicolumn{7}{|l|}{ Upper path } \\
\hline Numerical calculation & $2.18-0.18 r$ & $2.18+0.18 r$ & 2.15 & $0.248-0.023 r$ & $0.248+0.023 r$ & 0.245 \\
\hline Apex approximation & $2.09-0.16 r$ & $2.09+0.16 r$ & 2.08 & - & - & - \\
\hline \multicolumn{7}{|l|}{ Axial path } \\
\hline Numerical calculation $\left(\theta_{0}=+0.1^{\circ}\right)$ & $3.56-0.93 r$ & $3.56+0.93 r$ & 3.19 & $2.18-0.57 r$ & $2.18+0.57 r$ & 1.92 \\
\hline Numerical calculation $\left(\theta_{0}=-0.1^{\circ}\right)$ & $3.68-0.95 r$ & $3.68+0.95 r$ & 3.31 & $1.59-0.41 r$ & $1.59+0.41 r$ & 1.41 \\
\hline Axial approximation & $3.45-0.95 r$ & $3.45+0.95 r$ & 3.37 & - & - & - \\
\hline \multicolumn{7}{|l|}{ Lower path } \\
\hline Numerical calculation & $0.550-0.135 r$ & $0.550+0.135 r$ & 0.503 & $0.035-0.010 r$ & $0.035+0.010 r$ & 0.032 \\
\hline Approximation for $A^{-1}$ & - & - & - & $0.030-0.008 r$ & $0.030+0.008 r$ & 0.027 \\
\hline
\end{tabular}

ated. Table 3 gives the measured $\ln$-intensity fluctuations for both the lower and upper paths, and compares the lower path results to theoretical values obtained both by exact numerical calculation of the phase curvature, $A^{-1}$, and by use of the lower path approximation

$$
A^{-1}=x(R-x) / R
$$

The approximation of Eq. (57) is evidently quite good for this geometry, giving results within about $15 \%$ of the exact calculation. For the lower path the measured variances, $\left\langle\iota_{+} \iota_{+}\right\rangle$and $\left\langle\iota_{-} \iota_{-}\right\rangle$, can be

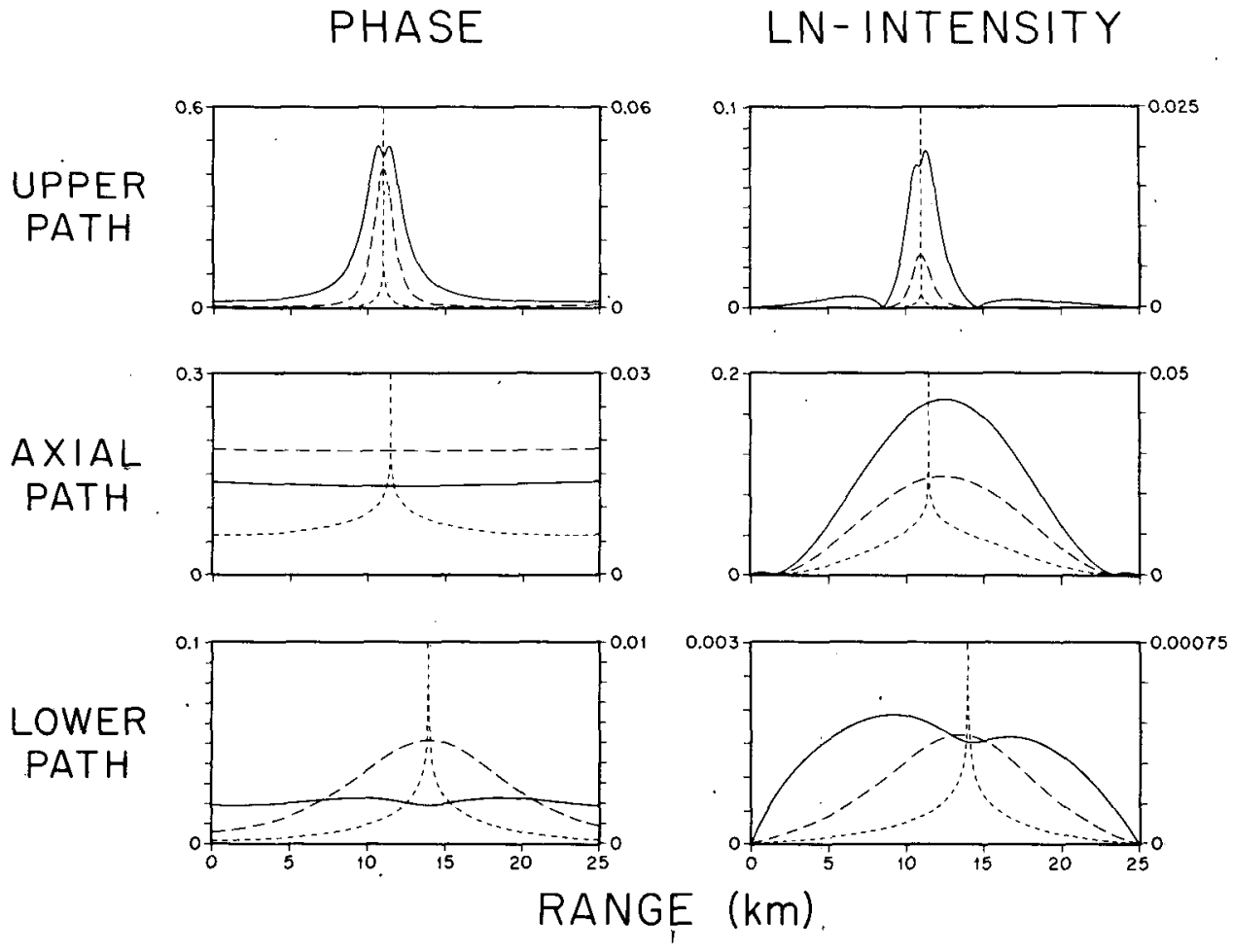

FIG. 2. Contributions to the phase and ln-intensity covariances given in Table 4 as a function of range (upper and lower ray paths are shown in Fig. 1). The integrands for $\Phi_{c c}, I_{c c}$ (solid, left scales) are much larger than the integrands for $\Phi_{u u}, I_{u u}$ (short dashes, right scales) or $\Phi_{c u}, I_{c u}$ (long dashes, right scales). The negative of $\Phi_{c u}$ and $I_{c u}$ for $r=1$ are plotted. Results are displayed for the axial path with $\dot{\theta}_{0}=+0.1^{\circ}$. Phase is in $\left(\mathrm{rad}^{2} \mathrm{~km}^{-1}\right)$; multiply ln-intensity by $(10 / \mathrm{ln} 10)^{2}$ to obtain $\left(\mathrm{dB}^{2} \mathrm{~km}^{-1}\right)$. 
reconciled with the predicted variances for a small value of $r$, but the cross-covariance, $\left\langle\iota_{+} \iota_{-}\right\rangle$, is much lower than predicted. The upper path variances are near the well-known theoretical value for the saturated regimes of 1.64 [corresponding to $(5.6 \mathrm{~dB})^{2}$ ]; the cross-covariance is low and similar to that measured for the lower path.

The disagreement between the measured and predicted cross-covariance is at best only suggestive due to the extremely short time series available. If one takes the discrepancy seriously, however, a plausible explanation is suggested by the similarity between the upper and lower path values of $\left\langle\iota_{+} \iota_{-}\right\rangle$. The upper path as computed for the mean soundspeed profile lies in the partially saturated regime, and is broken into a number of distinct Fermat paths (micromultipaths) by the internal wave perturbations. Interference effects among the micromultipaths are then important, and small changes in the sound-speed and/or current field can lead to large changes in the interference pattern (fades, for example). The lower path is close to the boundary between the unsaturated and partially saturated regimes $\left(\Phi^{2} \Lambda=1, \Lambda<1\right)$; the boundary region is not well understood, but it is possible that sporadic micromultipathing occurs and causes interference effects. The lower path was in fact found to be occasionally split into two distinct peaks.

At an acoustic frequency of $100 \mathrm{~Hz}$ both the upper and lower paths would be in the unsaturated regime. Table 4 summarizes the theoretical phase and lnintensity covariances at this frequency (for completeness, an axial path with the source at $z_{1}$ $=-0.700 \mathrm{~km}$ is included). Fig. 2 shows the contributions along the acoustic paths (Fig. 1) towards the various covariances. There are a number of interesting features.

The upper path cross-covariances, $\left\langle\phi_{+} \phi_{-}\right\rangle$and $\left\langle\iota_{+} \iota_{-}\right\rangle$, differ little from the variances for $r=0$ : current fluctuations are unimportant compared to sound speed fluctuations for the upper path, as expected. Further, the apex approximation is quite good for phase covariances along the upper path. All integrands are strongly peaked at the apex (Fig. 2).

The axial path with $\theta_{0}=+0.1^{\circ}$ is slightly over the boundary into the partially saturated regime (the ln-intensity variances exceed the saturated value of 1.64). The predicted In-intensity covariances for the axial path apparently are sensitive to small changes, however; the predictions for a ray with source angle $\theta_{0}=-0.1^{\circ}$ differ considerably from those for one with $\theta_{0}=+0.1^{\circ}$. The axial approximation for phase covariances is quite good.

The lower path cross-covariances, $\left\langle\phi_{+} \phi_{-}\right\rangle$and $\left\langle\iota_{+} \iota_{-}\right\rangle$, are smaller relative to the variances than was the case for the upper path: currents are relatively more important. The cross covariances are still predicted to be quite large, however. The lower path approximation for the phase curvature appears to be good.

\section{Measuring momentum flux}

An important and difficult problem is to measure the vertical flux of horizontal momentum. There have been some previous estimates [using rotary decomposition of repeated current profiles (Leaman, 1976)]. To make estimates useful one will want to impose the least restrictive assumptions on the internal wave field; so far there have been rather too many of these assumptions.

In the discussion so far we have considered only the mean products $\left\langle\phi_{+}(t) \phi_{+}(t)\right\rangle,\left\langle\phi_{-}(t) \phi_{-}(t)\right\rangle$, $\left\langle\phi_{+}(t) \phi_{-}(t)\right\rangle$ of the measured time series $\phi_{+}(t)$ and $\phi_{-}(t)$. The measurements can be extended to give the mean lagged products and their Fourier transforms:

$$
\left.\begin{array}{l}
\left\langle\phi_{+}(t) \phi_{+}(t-\tau)\right\rangle=\int d \omega P_{\phi_{+} \phi_{+}}(\omega) \cos \omega \tau \\
\left\langle\phi_{-}(t) \phi_{-}(t-\tau)\right\rangle=\int d \omega P_{\phi_{-} \phi_{-}}(\omega) \cos \omega \tau \\
\left\langle\phi_{+}(t) \phi_{-}(t-\tau)\right\rangle \\
=\int d \omega\left[P_{\phi_{+} \phi_{-}}(\omega) \cos \omega \tau+Q_{\phi_{+} \phi_{-}}(\omega) \sin \omega \tau\right]
\end{array}\right\} .
$$

$P_{a b}$ and $Q_{a b}$ are the cospectra and quadrature spectra, respectively, of any two time series $a(t)$, $b(t)$. When $a=b$, then $Q_{a a}=0$ and $P_{a a}$ is the same as the power spectrum. Further, $P_{a b}=P_{b a}$ and $Q_{a b}$ $=-Q_{b a}$. Eq. (30) is generalized to give

$$
\left.\begin{array}{c}
\left\langle\phi_{+}(t) \phi_{+}(t-\tau)\right\rangle=\cdots \int d x \\
\cdots \sum_{j} \cdots \int d \omega \cdots P_{\mu_{+} \mu_{+}}(\omega) \cos \omega \tau \\
\left\langle\phi_{-}(t) \phi_{-}(t-\tau)\right\rangle=\cdots \int d x \\
\ldots \sum_{j} \cdots \int d \omega \cdots P_{\mu_{-} \mu_{-}}(\omega) \cos \omega \tau \\
\left\langle\phi_{+}(t) \phi_{-}(t-\tau)\right\rangle \\
\left.\ldots \int_{j} d x \cdots \sum_{j} \cdots \int d \omega_{\mu_{+} \mu_{-}}(\omega) \cos \omega \tau+Q_{\mu_{+} \mu_{-}}(\omega) \sin \omega \tau\right]
\end{array}\right\}
$$


We need the expansions [using (4)]

$$
\begin{aligned}
& \left\langle\mu_{+}(t) \mu_{+}(t-\tau)\right\rangle \\
& =\left\langle\frac{\delta c(t)}{c} \frac{\delta c(t-\tau)}{c}\right\rangle+\left\langle\frac{u(t)}{c} \frac{u(t-\tau)}{c}\right\rangle \\
& \quad+\left\langle\frac{\delta c(t)}{c} \frac{u(t-\tau)}{c}\right\rangle+\left\langle\frac{u(t)}{c} \frac{\delta c(t-\tau)}{c}\right\rangle \\
& \left\langle\mu_{-}(t) \mu_{-}(t-\tau)\right\rangle=\langle\rangle+\langle\rangle-\langle\rangle-\langle\rangle, \\
& \left\langle\mu_{+}(t) \mu_{-}(t-\tau)\right\rangle=\langle\rangle-\langle\rangle-\langle\rangle+\langle\rangle .
\end{aligned}
$$

Performing the Fourier transform, and equating terms in $\cos \omega \tau$, and terms in $\sin \omega \tau$, gives

$$
\left.\begin{array}{l}
P_{\mu_{+} \mu_{+}}=P_{c c}+P_{u u}+2 P_{c u} \\
P_{\mu_{-} \mu_{-}}=P_{c c}+P_{u u}-2 P_{c u} \\
P_{\mu_{+} \mu_{-}}=P_{c c}-P_{u u} \\
Q_{\mu_{+} \mu_{-}}=-2 Q_{c u}
\end{array}\right\}
$$

These are substituted on the right hand side of (59); for the left-hand side we use (58). Equating the $\omega$ integrands leads to

$$
\left[\begin{array}{c}
P_{\phi_{+} \phi_{+}} \\
P_{\phi_{-} \phi_{-}} \\
P_{\phi_{+} \phi_{-}} \\
Q_{\phi_{+} \phi_{-}}
\end{array}\right]=\cdots \int d x \cdots \sum_{j} \frac{1}{j} \frac{\omega_{i n}}{\left(\omega^{2}-\omega_{L}^{2}\right)^{1 / 2}} \times\left[\begin{array}{c}
P_{c c}+P_{u u}+2 P_{c u} \\
P_{c c}+P_{u u}-2 P_{c u} \\
P_{c c}-P_{u u} \\
-2 Q_{c u}
\end{array}\right]
$$

Finally, we require the relation between the acoustic spectra and the spectrum of vertical momentum flux, that is the cospectrum of $w u=\dot{\zeta} u$. For a stationary process $P_{\zeta u}=\omega Q_{\zeta u}$; from (5)

$$
P_{\dot{c} u}=\omega Q_{c u}=24.5 g^{-1} n^{2}(z) P_{w u} .
$$

By various combinations of (61) we obtain our final result

$$
\left[\begin{array}{c}
1 / 4\left(P_{\phi_{+} \phi_{+}}+P_{\phi_{-} \phi_{-}}+2 P_{\phi_{+} \phi_{-}}\right) \\
1 / 4\left(P_{\phi_{+} \phi_{+}}+P_{\phi_{-} \phi_{-}}-2 P_{\phi_{+} \phi_{-}}\right) \\
-1 / 2 \omega Q_{\phi_{+} \phi_{-}}
\end{array}\right]=\frac{2}{\pi} q^{2} b \frac{n_{0}}{\omega_{i n}} \int d x \sec ^{2} \theta \sum_{j} \frac{1}{j} \frac{\omega_{i n}}{\left(\omega^{2}-\omega_{L}^{2}\right)^{1 / 2}} \times\left[\begin{array}{c}
P_{c c} \\
P_{u u} \\
P_{c u}
\end{array}\right]
$$

The important result is that the spectrum of momentum flux is related to the quadrature spectrum of reciprocal travel times.

The integral equations are all of the form

$$
\begin{aligned}
F(\omega)=\sum_{j} \frac{1}{j} \int d x \sec ^{2} \theta \frac{\omega_{\text {in }}}{\left(\omega^{2}-\omega_{L}^{2}\right)^{1 / 2}} \\
\times P\left[\omega, j, \alpha_{0}(\omega, x) ; z(x)\right],
\end{aligned}
$$

where

$$
\tan \alpha_{0}=-\frac{\left(\omega^{2}-\omega_{L}^{2}\right)^{1 / 2}}{n \tan \theta}
$$

by stationary phase considerations. The ray integrals $\int d x$ are accordingly constrained to such portions of the ray for which

$$
\omega_{\text {in }}^{2}+n^{2} \tan ^{2} \theta \equiv \omega_{L}^{2} \leqslant \omega^{2} \leqslant n^{2} .
$$

This constraint is illustrated in Fig. 5 of Munk and Zachariasen (1976). If the integral equations (63) can be solved for $P$, then we have a way of obtaining the oceanographically interesting spectra of $\left\langle\zeta^{2}\right\rangle$, $\left\langle u^{2}\right\rangle$ and $\langle w u\rangle$ characterizing the internal wave field in terms of measured spectra $P_{\phi_{+} \phi_{+}}, P_{\phi_{-} \phi_{-}}, P_{\phi_{+} \phi_{-}}$, $Q_{\phi_{+} \phi_{-}}$for reciprocal acoustic transmissions.
We change to a depth integration $\int d x \cdots=\int d z$ $\times \cot \theta \cdots$ and write Eq. (63) in the form

$F(\omega)=\sum_{j} j^{-1} \int d z K(\omega, z) G\left[\omega, j, \alpha_{0}(\omega, z) ; z\right]$

with

$$
K(\omega, z)=\frac{1}{\sin \theta \cos \theta} \frac{\omega_{i n}}{\left(\omega^{2}-\omega_{L}^{2}\right)^{1 / 2}},
$$

subject to the constraint $(65) . F(\omega)$ is a function measured by the reciprocal transmissions, $K(\omega, \dot{z})$ and $\alpha_{0}(\omega, z)$ are determined from the profiles $c(z)$ and $n(z)$ and presumed as known, and $G\left(\omega, j, \alpha_{0}\right.$; $z$ ) is an unknown function of all of its arguments. For several resolvable multipaths, $i=1,2, \ldots$, with ray inclinations $\theta_{i}(z)$, each yielding a measured spectrum $F_{i}(\omega)$, we have

$$
\begin{gathered}
F_{i}(\omega)=\sum_{j} j^{-1} \int d z K_{i}(\omega, z) G\left[\omega, j, \alpha_{0 i}(\omega, z) ; z\right], \\
i=1,2, \ldots
\end{gathered}
$$

The inversion of these integral equations is a problem of inverse theory, and one can use the powerful 

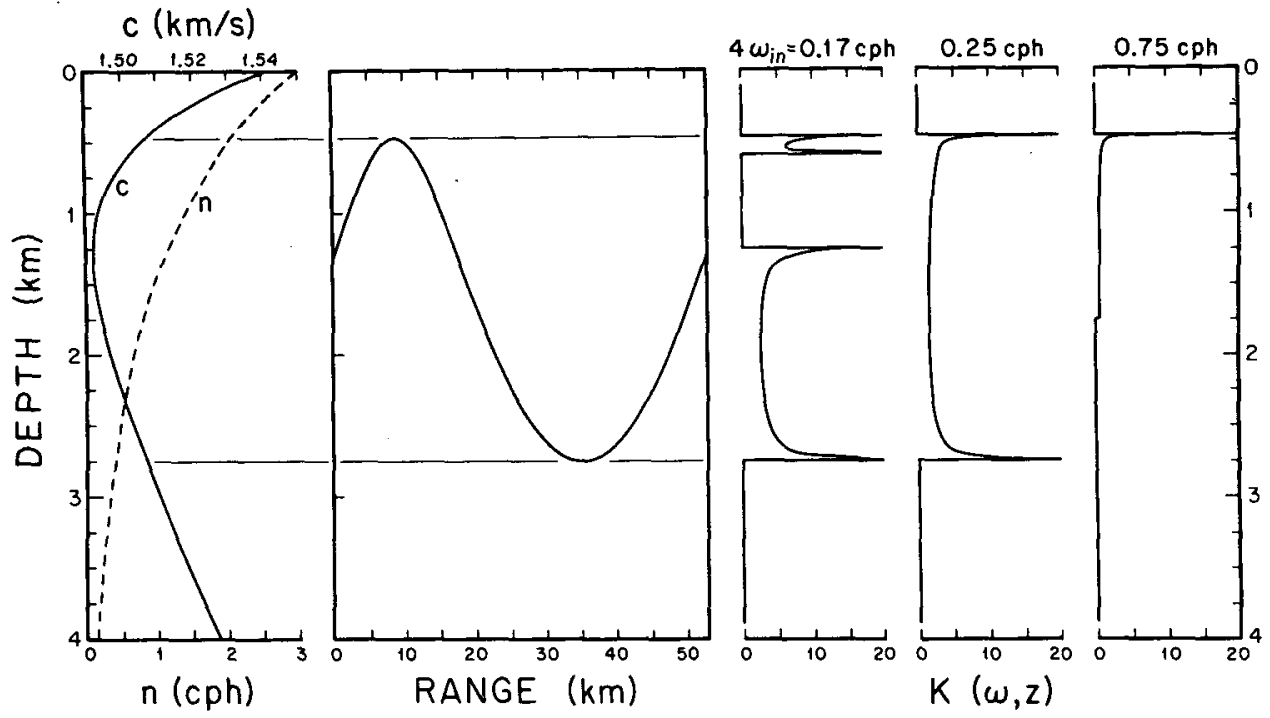

FIG. 3. The kernel $K(\omega, z)$ at three selected frequencies, for a canonical sound channel (left) and a particular ray path (center) which crosses the sound channel axis with an inclination of $8^{\circ}$.

apparatus that has been developed for such purposes. However, the constraints on $\alpha$ and $z$ will make it impossible to evaluate $P$ in its generality. One may hope that the $j$-dependence can be factored, and that the dependence on direction $\alpha$ is weak, in which case the integral equations reduce to the form

$F_{i}(\omega)=\left\langle j^{-1}\right\rangle \int d z K_{i}(\omega, z) G(\omega, z), \quad i=1,2, \ldots$

As a practical matter, a numerical inversion of even this simplified equation raises all kinds of questions of accuracy, resolution and uniqueness. We think that these should be examined in the context of a specific experiment, rather than at this time.

The behavior of the weighting kernels $K_{i}(\omega, z)$ is crucial to the application of the proposed method. For illustration in Fig. 3 we have chosen a typical fairly steep ray in a canonical sound channel (Munk, 1974), but even for this smooth channel the kernel is complex. First of all, there are singularities associated at the turning points $\hat{z}$, when $\omega<n(\hat{z})$. [This excludes a singularity at the low turning point for the case $\omega=0.75 \mathrm{cph}$ which has a forbidden region $(n<\omega)$ beneath $1.8 \mathrm{~km}$.] Then there are singularities associated with $\left(\omega^{2}-\omega_{L}^{2}\right)^{-1 / 2}$ unless $\omega>\omega_{L}$ everywhere along the ray; these occur at $0.65 \mathrm{~km}$ and $1.20 \mathrm{~km}$ for the case $\omega=0.17 \mathrm{cph}$, but not at the higher frequencies. But all singularities are integrable, and there is a significant contribution along the entire (non-forbidden) ray paths.

Acknowledgments. Some of this material was contained in JASON Summer Report 1977-21. We are indebted to the Office of Naval Research for supporting this investigation.

\section{APPENDIX A}

An Integral Involving $\boldsymbol{\Omega}_{u u}$

$\Omega_{u u}$ [Eq. (46)] diverges as $D \rightarrow 1$. Set

$$
\Delta^{2}=D^{2}-1=\left(n / \omega_{i n}\right)^{2} \tan ^{2} \theta
$$

Then the integral approaches

$$
\Omega_{u u}=\frac{4}{\pi} \ln \frac{2}{\Delta} .
$$

We can express $\Delta$ as a function of range. Let source and receiver be on the axis at $x=\mp 1 / 2 R$. Then with $\delta z=A \cos \pi x / R$ designating the distance of the ray above the sound axis, we find (Munk, 1974) $A$ $=-3 / 4 \pi B\left(R-R_{0}\right) / R_{0}$ where $R_{0}=1 / 2 \pi B \epsilon^{-1 / 2}=21$ $\mathrm{km}$ refers to the axial loop $\left(R<R_{0}\right.$ for upward loops). It follows that

$$
\begin{aligned}
& \theta=\frac{\delta z}{\delta x}=\frac{3 \pi^{2}}{4} \frac{B}{R} \frac{R-R_{0}}{R_{0}} \sin \frac{\pi x}{R}, \\
& \Delta \approx \frac{n_{1} \theta}{\omega_{i n}}=\frac{3 \pi^{2}}{4} \frac{n_{1}}{\omega_{i n}} \frac{B}{R} \frac{R-R_{0}}{R_{0}} \sin \frac{\pi x}{R} .
\end{aligned}
$$

Setting $B=1 \mathrm{~km}, n_{1}=1.9 \times 10^{-3} \mathrm{~s}^{-1}, \omega_{i n}=7.3$ $\times 10^{-5} \mathrm{~s}^{-1}, R_{0}=21 \mathrm{~km}$, we have

$$
\Delta=9.2 \frac{R-R_{0}}{R_{0}} \sin \frac{\pi x}{R} .
$$




\section{We now require}

$$
\begin{aligned}
& R^{-1} \int_{-(1 / 2) R}^{(1 / 2) R} d x \Omega_{m u} \\
& =\frac{4}{\pi R} \int_{-(1 / 2) R}^{(1 / 2) R} d x\left[\ln \frac{2 R_{0}}{9.2\left|R-R_{0}\right|}+\ln 2\right] \\
& =\frac{4}{\pi} \ln \left[\frac{4 R_{0}}{9.2\left|R-R_{0}\right|}\right] .
\end{aligned}
$$

Thus even if we are within $0.1 \mathrm{~km}$ of the convergence distance, $\left|R-R_{0}\right|=0.1 \mathrm{~km}$, the effect of currents is still relatively small. So the logarithmic singularity is of no practical interest.

\section{REFERENCES}

Briscoe, M. G., 1975: Preliminary results from the trimoored internal wave experiment (IWEX). J. Geophys. Res., 80, $3872-3884$.

Flatté, S. M., Ed., R. Dashen, W. H. Munk, K. M. Watson and F. Zachariasen, 1979: Sound Transmission Through a Fluctuating Ocean. Cambridge University Press, 299 pp.

Flatté, S. M., R. Leung and S. Y. Lee, 1980: Frequency spectra of acoustic fluctuations caused by internal waves and other finestructure. J. Acoust. Soc. Amer., 68, 1773-1779.

Garrett, C. J. R., and W. H. Munk, 1972: Space-time scales of internal waves. Geophys. Fluid Dyn., 2, 225-264.

- and W. H. Munk, 1975: Space-time scales of internal waves: a progress report. J. Geophys. Res., 80, 291-297.

Leaman, K. D., 1976: Observations of vertical polarization and energy flux of near-inertial waves. J. Phys. Oceanogr., 6, 894-908.

McComas, C. H., 1977: Equilibrium mechanism within the oceanic internal wave field. J. Phys. Oceanogr., 7, 836-845.

Müller, P., D. J. Olbers, and J. Willebrand, 1978: The IWEX spectrum. J. Geophys. Res., 83, 479-500.

Munk, W., 1974: Sound channel in an exponentially stratified ocean: with application to SOFAR. J. Acoust. Soc. Amer., $55,220-226$.

_- 1980: Internal waves and small-scale processes. The Ocean Environment, C. Wunsch and B. Warren, Eds. The MIT Press, 264-291.

—, and F. Zachariasen, 1976: Sound propagation through a fluctuating stratified ocean: theory and observation. $J$. Acoust. Soc. Amer., 59, 818-838.

Pomphrey, N., J. D. Meiss and K. M. Watson, 1980: Description of nonlinear internal wave interactions using Langevin methods. J. Geophys. Res., 85, 1085-1094.

Worcester, P. F., 1977: Reciprocal acoustic transmission in a midocean environment. J. Acoust. Soc. Amer., 62, 895-905.

_, 1979: Reciprocal acoustic transmission in a midocean environment: Fluctuations. J. Acoust. Soc. Amer., 66, 11731181 . 